\title{
DiaTOMÁCEAS EM SEDIMENTOS SUPERFICIAIS NA PLANÍCIE DE MARÉ da Praia de Itupanema, estado do Pará, Amazônia ${ }^{1}$
}

\author{
Fábio Campos Pamplona Ribeiro ${ }^{2,4}$, \\ Cristina do Socorro Fernandes de Senna ${ }^{2} \&$ Lezilda Carvalho Torgan ${ }^{3}$
}

\begin{abstract}
RESUMO
(Diatomáceas em sedimentos superficiais na planície de maré da Praia de Itupanema, estado do Pará, Amazônia) O objetivo deste artigo é avaliar a composição, abundância e riqueza das diatomáceas presentes na zona intermaré da Praia de Itupanema e relacionar esses atributos à hidrodinâmica atual, evidenciada pela textura do sedimento. Foram coletadas 10 amostras em duas transecções perpendiculares, em intervalos de $5 \mathrm{~m}$ nas coordenadas 01³1'03'S e 48 43'28'W em março de 2006, sendo analisadas segundo as técnicas convencionais no estudo de diatomáceas. Os resultados mostraram a ocorrência de 58 espécies, 82,75\% bentônicas/epifíticas e 17,25\% planctônicas, comuns em águas doces a oligo-mesohalinas, as mais abundantes e freqüentes em ordem decrescente foram Staurosirella pinnata, Aulacoseira granulata, Thalassiosira sp., Staurosira obtusa, Encyonema silesiacum, Gomphonema parvulum, Planothidium lanceolatum, Thalassiosira eccentrica, Cyclotella meneghiniana, Encyonema minutum, Nitzschia amphibia e Frustulia sp. Os dados também revelaram a influência da relação areia/argila sobre a composição e a abundância das diatomáceas, o que pode explicar a prevalência local de agrupamentos de espécies ora planctônicas, ora bentônicas na zona intermaré, como resposta aos processos hidrodinâmicos atuais. Há de se salientar a provável contribuição da vegetação herbácea, atenuando a energia das ondas e marés, retendo os sedimentos finos argilosos, promovendo assim, a redução de processos de abrasão na zona intermaré e a conseqüente preservação dos táxons planctônicos.
\end{abstract}

Palavras-chave: diatomáceas, hidrodinâmica, estuário.

\section{Abstract}

(Diatoms on surface sediments of tidal plain, Itupanema Beach, Pará State, Amazon) This paper aim evaluate the composition, abundance and richness of diatoms and to relate theses attributes to actual hydrodynamic processes evidenced by sedimentary texture on surface sediments located on intertidal zone of Itupanema Beach, situated on estuarine continental sector of the Pará State, Amazon. The 10 samples were collected in perpendicular transects within 5 m intervals on coordinates $01^{\circ} 31^{\prime} 03^{\prime}$ 'S and 48 43 '28' 'W in 2006, March and were applied diatoms analysis following standard techniques. The results showed 58 species, being $82,75 \%$ benthic/epiphytic and $17,25 \%$ planktonic species, occurring commonly in fresh to oligo-mesohaline waters, the more abundant and frequent species in decrescent order were Staurosirella pinnata, Aulacoseira granulata, Thalassiosira sp, Staurosira obtusa, Encyonema silesiacum, Gomphonema parvulum, Planothidium lanceolatum, Thalassiosira eccentrica, Cyclotella meneghiniana, Encyonema minutum, Nitzschia amphibia and Frustulia sp. The data also revealed that the sedimentary ratio sand/ clay was influenced by actual hydrodynamic processes, probably determining the local prevalence of planktonic species groups or benthic species groups on the intertidal zone, in terms of the composition and the abundance of diatoms species. The aquatic herbaceous vegetation had a possible role on the reduction the both energy waves and tidal current velocities, and the retention of muddy sediments, with the reduction of abrasion processes in the intertidal zone and consequently preserving planktonic taxa.

Key words: diatoms, hydrodynamic, estuary

\section{INTRODUÇÃO}

Os estuários apresentam alta variabilidade na salinidade, regime de deposição sedimentar, correntes, turbidez e biogeoquímica dos sedimentos (Cooper 1999). Dentre os ambientes presentes nos estuários, as áreas de intermarés são altamente dinâmicas, sendo constantemente influenciadas por níveis de energia local e

\footnotetext{
Artigo recebido em 06/2007. Aceito para publicação em 01/2008.

${ }^{1}$ Parte da Dissertação de Mestrado do primeiro autor, apresentada ao Curso de Botânica Tropical-MPEG/UFRA - Apoio financeiro: Companhia Vale do Rio Doce - Programa de Arqueologia Preventiva na área do Projeto Bauxita Paragominas/PA. ${ }^{2}$ Laboratório de Palinologia e Paleoecologia da Amazônia-LAPPAM, Coordenação de Ciências da Terra e Ecologia. Museu Paraense Emílio Goeldi/MCT. C.P. 399, 66040-170, Belém, PA, Brasil.

${ }^{3}$ Museu de Ciências Naturais - Fundação Zoobotânica do Rio Grande do Sul. C.P. 1188, Porto Alegre, RS, Brasil.

${ }^{4}$ Autor para correspondência: fbpamplona@yahoo.com.br
} 
especialmente, no caso de planícies arenosas com alta energia, exibem uma estrutura governada por repetidos processos de erosão e redeposição. As diatomáceas residentes nestas áreas são adaptadas a essas condições e possuem mecanismos para superar as situações adversas (Mitbavkar \& Anil 2002).

A abundância algal e a composição das espécies são também controlados pelo tempo disponível para colonização do substrato e pela microtopografia da superfície (Burkholder 1996). As características físicas do substrato como a textura e a composição, somadas ao tempo de permanência deste, são os maiores fatores influenciando a colonização algal, especialmente em águas correntes, ou em zonas de quebra das ondas em lagos (Burkholder 1996).

As pequenas rochas e sedimentos movidos pela ação de ondas e correntes, com taxas de retrabalhamento altamente variáveis, influenciadas pelo vento, chuvas ou eventos de inundação, podem selecionar microalgas móveis tais como as diatomáceas, em oposição a populações de macroalgas sésseis, mais facilmente soterradas e esmagadas (Burkholder 1996).

O sedimento superfícial $(0-1 \mathrm{~cm})$ representa uma amostra integrada temporariamente, por táxons acumulados em um passado muito recente. A mesma amostra integra-se também espacialmente, pois incorpora táxons derivados de uma variedade de diferentes comunidades fonte, a partir de uma completa extensão de hábitos disponíveis, quais sejam, a comunidade planctônica que se encontra suspensa na água, a epifítica que cresce fixada sobre vegetação aquática e comunidades epipélica e episâmica, que vivem na superfície do sedimento lamoso e arenoso, respectivamente. Em adição, o incremento de diatomáceas no sedimento representa uma mistura de comunidades sucessionais, cuja proporção nos sedimentos, está relacionada à produtividade (Fritz et al. 1999).

As assembléias de diatomáceas têm sido utilizadas para inferir variáveis ambientais em séries temporais de calibração, importantes para interpretações de eventos paleoambientais, paleoclimáticos e paleohidrológicos, através da análise de sedimentos superficiais.
Diversas variáveis como o $\mathrm{pH}$, nutrientes, salinidade, luz, profundidade, tipo de sedimento etc., são mensuradas através de um gradiente, assim como a composição de táxons preservados nos sedimentos ao longo deste (Hall \& Smol 1999). Através de análises estatísticas, é possível quantificar a resposta de cada táxon, em relação a uma dada variável ambiental. Assim, as condições ambientais podem ser calculadas através da composição de espécies nas amostras, baseadas em estimativas quantitativas das respostas das espécies derivadas das séries de calibração (Fritz et al. 1999).

Os trabalhos sobre análise de diatomáceas em sedimentos superficiais são ainda escassos no Brasil, concentrados em manguezais, praias e lagoas costeiras, entretanto, muitos deles realizaram apenas levantamentos da flora algal (Silva \& Cimardi 1989; Callegaro \& Lobo 1990; Felício-Fernandes \& Souza-Mosimann 1994; Garcia-Baptista 1996; Sylvestre et al. 2001; Pires \& Lacerda 2004; Gomes et al. 2005; Ribeiro 2007; Ribeiro et al. 2007).

A pesquisa pioneira de Silva \& Cimardi (1989) foi realizada no manguezal do rio Ratones, Santa Catarina, onde as diatomáceas epipélicas foram separadas do sedimento, utilizando armadilhas confeccionadas com tecidos de celulose. O gênero Nitzschia foi melhor representado com seis espécies (Nitzschia closterium, N. fasciculata, N. granulata, $N$. obtusa var. scalpelliformis, $N$. panduriformis var. minor e $N$. sigma var. rigida).

Callegaro \& Lobo (1990) estudaram as comunidades de diatomáceas de sedimentos superficiais ao longo de depósitos de turfa da fazenda Águas Claras, planície costeira do Rio Grande do Sul, onde ocorreram duas associações de diatomáceas, com Eunotia lineolata var. lineolata e Pinnularia maior var. maior ( $1^{\mathrm{a}}$ associação) e Pinnularia microstauron var. microstauron, Frustulia rhomboides var. saxonica ( $2^{\text {a }}$ associação). As preferências ecológicas das espécies indicaram a existência de um ambiente lêntico, oligoalino e ácido.

A pesquisa de Felício-Fernandes \& SouzaMosimann (1994) foi realizada em sedimentos do manguezal de Itacorubi, Santa Catarina, 
onde os autores identificaram 121 táxons, destacando-se os gêneros Nitzschia (24 táxons) e Navicula (15 táxons).

Garcia-Baptista (1996) estudou a distribuição das microalgas psâmicas em sedimentos superficiais da praia Azul, RS, onde as diatomáceas destacaram-se, com 59,8\% do total de espécies, sendo o grupo mais importante também, em termos de abundância, onde a descontinuidade da distribuição das comunidades foi influenciada pela condutividade, relacionada à topografia do terreno.

Sylvestre et al. (2001) identificaram 156 diatomáceas em 75 amostras de sedimentos superficiais provenientes da lagoa hipersalina Araruama, RJ, onde as assembléias mostraram táxons bentônicos holo-eurihalinos e eurihalinos marinhos, principalmente, com a dominância das espécies Cocconeis plancetula var. euglypta, Catenula adhaerens e Cocconeis diminuta. Contudo, em algumas áreas amostradas, as assembléias continham táxons específicos, com a sua distribuição influenciada por variações na batimetria, relacionadas ainda a variação da salinidade, tendo em vista os aportes do oceano, rios adjacentes à lagoa e preciptações.

Pires \& Lacerda (2004) caracterizaram a flora de diatomáceas de um biofilme formado na interface sedimento-água do manguezal de Coroa Grande, Baía de Sepetiba, RJ, mostrando que a comunidade das microalgas constituida pelas espécies Auliscus coelatus, Actinoptychus undulatus, Biddulphia sp., Cocconeis scutellum, Cyclotella stylorum, Fragilaria sp., Melosira nummuloides, Navicula lanceolata, Thalassionema sp. e Thalassiosira sp. teve efetiva participação da nos processos biogeoquímicos.

Gomes et al. (2005) analizaram a distribuição de diatomáceas em sedimentos superficiais em função da profundidade na Lagoa do Boqueirão, ao norte do estado do Rio Grande do Norte, inserida em uma planície costeira com sistema dulciaquícola, visando a calibração de séries temporais para possíveis reconstituições paleoambientais. Foram identificadas 61 espécies, onde Mastogloia smithii var. lacustris foi a mais abundante, em trechos mais profundos do sistema, enquanto Staurosirella pinnata e Nitzschia amphibia atingiram os maiores valores quantitativos, em trechos mais rasos.

No estuário amazônico, os trabalhos com diatomáceas em sedimentos superficiais estão no início, destacando-se Ribeiro (2007), que analisou diatomáceas em amostras sedimentares superficiais da Praia de Itupanema, município de Barcarena, Pará, identificando 58 espécies, sendo as mais abundantes Staurosirella pinnata, Aulacoseira granulata, Thalassiosira sp., Staurosira obtusa, Encyonema silesiacum, Gomphonema parvulum, Planothidium lanceolatum, Thalassiosira eccentrica, Cyclotella meneghiniana, Encyonema minuta, Nitzschia amphibia e Frustulia sp.

Ribeiro et al. (2007) analisaram diferentes substratos da planície costeira da margem leste da Ilha de Marajó, município de Soure, PA, onde a maior riqueza nas assembléias diatomológicas foi encontrada na praia do Garrote (81 espécies), provavelmente devido ao aporte de água doce do rio Paracauari, seguida pela Praia do Cajuúna (69 espécies), com maior influência marinho/ estuarina e por último, a Fazenda Bom Jesus (49 espécies), cuja menor riqueza pode estar associada à baixa frequiência de inundação pelas marés, uma vez que apresenta dominância de abundantes táxons continentais.

Este artigo tem como objetivo o estudo da composição, abundância e riqueza de diatomáceas, relacionando os resultados com os processos hidrodinâmicos atuais, evidenciados pela textura dos sedimentos superficiais da zona intermaré da praia de Itupanema, Setor Continental Estuarino do estado do Pará, contribuindo para o conhecimento da flora diatomológica em sedimentos amazônicos, podendo ser aplicada no estudo de séries temporais sedimentares, úteis às reconstituições paleoambientais e paleohidrológicas do período Holoceno.

\section{Material e Métodos \\ Caracterização da área de estudo}

O estuário do rio Pará, com $300 \mathrm{~km}$ de extensão, inicia-se na baía da Bocas, no município de Breves, prosseguindo pelo rio 
Pará, que recebe toda a massa de águas do rio Tocantins e onde insere-se a praia de Itupanema. Inclui também a baía do Guajará, que recebe a embocadura dos rios Guamá/Moju/Acará/ Capim, em frente da cidade de Belém, passando à alongada baía de Marajó (Ab’ Saber 2006).

A praia de Itupanema faz parte do município de Barcarena, localizada no nordeste do Estado do Pará, limitando-se ao norte e a leste com a Baía de Marajó, ao sul com os municípios de Abaetetuba, Moju e Acará e a oeste com a Baía de Guajará (Oliveira 2002).

A área de estudo integra o setor continental estuarino (Alves et al. 2005), em termos fisiográficos, com sucessivos promontórios e enseadas (Farias 2004, apud Alves et al. 2005), cujas praias arenosas são curtas e estreitas, reflectivas e intermediárias e as várzeas de maré holocênicas são ligadas ao estuário do rio Pará e falésias do Grupo Barreiras (IDESP 1984, apud Oliveira 2002).

O clima da microregião é do tipo Afi de Köppen, com pluviosidade anual média superior a $2.000 \mathrm{~mm}$. O período chuvoso estende-se de dezembro a maio, enquanto no período seco, de junho a novembro, os totais pluviométricos caem pela metade (IDESP 1983, apud Oliveira 2002).

A geologia da região é representada por depósitos siliciclásticos (conglomerado, arenito, argilito) continentais da formação Barreiras, pouco ou mal selecionados de idade PlioPleistoceno, pertencentes ao Planalto Baixo Costeiro, enquanto os depósitos aluvionares recentes, constituídos por cascalhos, areias e argilas inconsolidadas do Holoceno integram a planície Flúvio-Marinha (Oliveira 2002). A Floresta Ombrófila Densa e a Floresta Aluvial compõem o revestimento florístico, entretanto, florestas secundárias também ocorrem atualmente, nas áreas de terra firme (Brasil 1974, apud Oliveira 2002).

Foram observados em campo, restos de troncos e raízes de árvores de várzea em posição de vida como Pterocarpus santalinoides L Her' DC. e Mauritia flexuosa L., Machaerium lunatum (L.f.) Ducke e Phthirusa paniculata (Kunth) J. F. Macbr., além de árvores de mangue
Rhizophora sp. e Avicennia germinans L. Dentre as espécies herbáceas que colonizam o substrato registrou-se Eleocharis intersticta (Vahl.) Roem. \& Schul., Eleocharis caribaea (Rottb.) Blacke, Cyperus luzulae (L.) Rottb. ex Retz., Cyperus comosus Poir. e Crenea maritima Aubl.

\section{Metodologia de campo}

Foram coletadas 10 amostras sedimentares superficiais, com um espaçamento de cinco metros, na porção intermaré da praia de Itupanema (01 32'03''S e 48 43'28'W), em março de 2006, ao longo de duas transecções ortogonais (Fig. 1). A primeira, disposta transversalmente à linha de praia, onde coletou-se as amostras de 1 a 5 , enquanto a segunda, disposta paralelamente à linha de praia, com a coleta das amostras 6 a 10.

A descrição dos sedimentos baseou-se na textura, estrutura e consistência (Lemos \& Santos 1996) e na cor (Munsell 1975). Houve variação na textura e na presença de restos vegetais e fragmentos de raízes, que integram boa parte da matéria orgânica do material coletado (Tab. 1).

\section{Metodologia de análise}

A análise diatomológica foi realizada em $2 \mathrm{~cm}^{3}$ de sedimentos, retirados de cada amostra, processadas de acordo com Moro \& Santi (1999). Foram montadas três lâminas permanentes com Bálsamo do Canadá (IR=1,54), analisadas em microscópio óptico Zeiss, objetiva de 100X (imersão) e em microscópio eletrônico de varredura Zeiss, modelo LEO $1450 \mathrm{VP}$, no Laboratório de Microscopia Eletrônica de Varredura do Museu Paraense Emílio Goeldi - LABMEV.

Após o término do trabalho, as lâminas foram depositadas na coleção de referência de diatomáceas da Amazônia do Laboratório de Palinologia e Paleoecologia da Amazônia (LAPPAM), Coordenação de Ciência da Terra e Ecologia/Museu Paraense Emílio Goeldi.

A classificação das espécies obedeceu ao sistema de Round et al. (1990). As informações sobre o hábito das espécies foram obtidas no catálogo de Moro \& Fürstengerger (1997). 


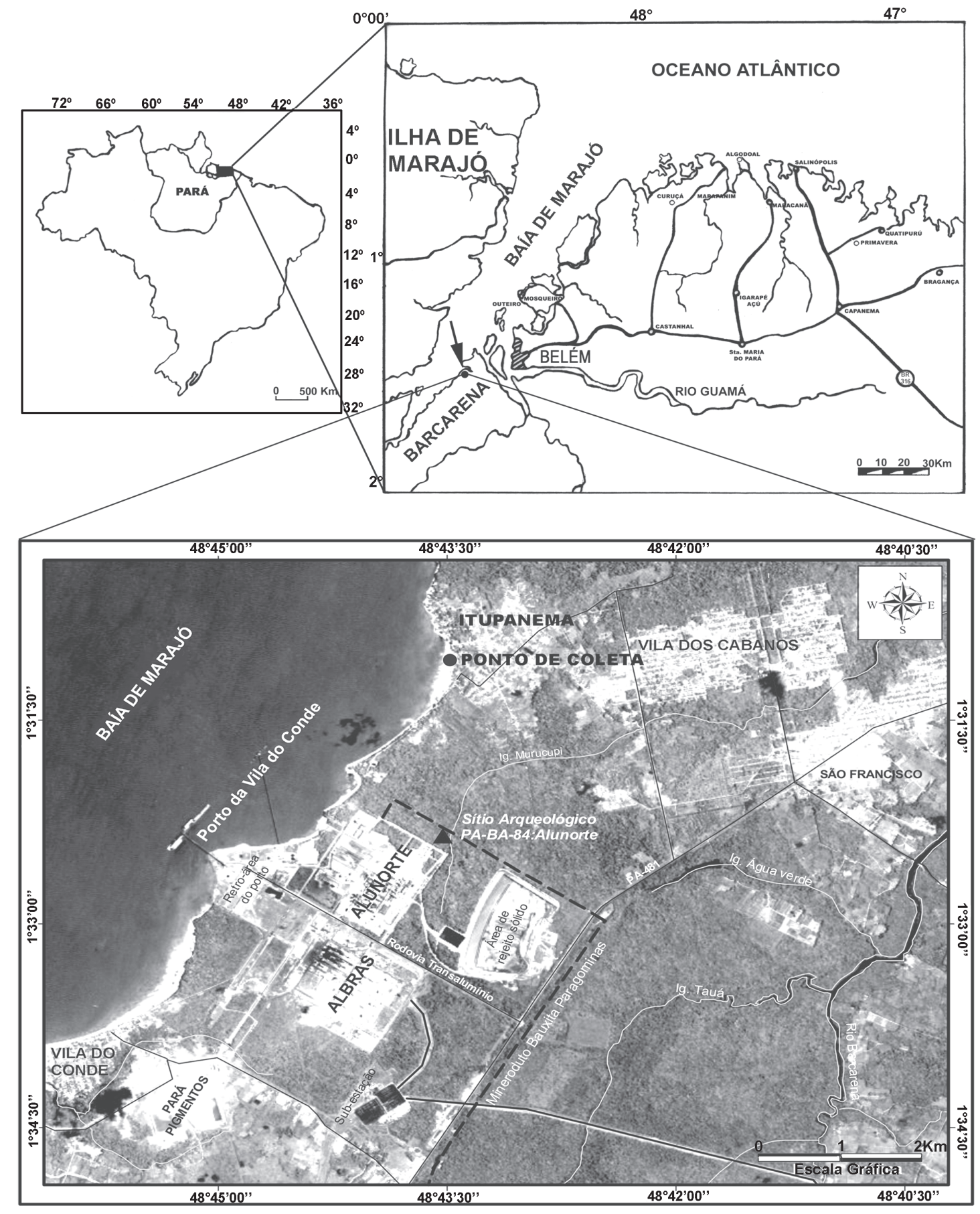

Figura 1 - Mapa de localização da área de estudo, na praia de Itupanema, Barcarena, Pará. Fonte: Lopes (2005). 
Tabela 1 - Peso, coloração e textura das amostras sedimentares de superfície coletados na praia de Itupanema, Barcarena, Pará.

\begin{tabular}{cccc}
\hline Amostras & Peso $($ g) & Cor & Textura \\
\hline 1 & 2,4856 & 7,5 YR $7 / 2$ & arenosa \\
2 & 2,7246 & 2,5 Y 5/4 a 10YR 5/4 & argilo-arenosa \\
3 & 4,0262 & 10 YR 5/6 & arenosa \\
4 & 3,4163 & 10 YR 4/4 & argilo-arenosa \\
5 & 2,8266 & 10 YR 3/3 & argilosa \\
6 & 2,4272 & 10 YR 4/2 & argilo-arenosa \\
7 & 3,0312 & 10 YR 4/2 & argilo-arenosa \\
8 & 2,6883 & 10 YR 3/3 a 3/4 & argilo-arenosa \\
9 & 2,3512 & 10 YR 5/4 & argilo-arenosa \\
10 & 3,5421 & 10 YR 5/6 & arenosa \\
\hline
\end{tabular}

A análise da estrutura da comunidade de diatomáceas em sedimentos, em resposta aos processos hidrodinâmicos atuais, baseou-se no conjunto de espécies mais representativas, que atingiram numericamente valores $>70 \%$ da abundância total.

A abundância relativa foi calculada a partir da contagem de 300 valvas, obtendo-se o número de indivíduos/espécie, enquanto para a frequiência de ocorrência utilizou-se a relação entre o número de amostras, nas quais cada espécie ocorre e o número total de amostras analisadas.

A comparação entre as amostras foi feita através da análise de agrupamento, baseada nos dados de presença e abundância de espécies, empregando-se o método de classificação hierárquico, com a medida de dissimilaridade de Bray-Curtis, que nãoé sensível aos zeros na matriz, dando maior peso às espécies dominantes. A ligação média ou UPGMA (Unweighted Pair Group Method with Arithmetic Mean) foi utilizada como algoritmo de ligação.

A técnica de ordenamento Análise de Correspondência (AC) foi também aplicada aos dados de abundância relativa das espécies, para a confirmação do padrão observado na Análise de Agrupamento e sua relação com a textura das amostras sedimentares. Para os dois tipos de análises, calculadas através do programa
PAST versão 1.4., foram utilizadas apenas as espécies que alcançaram valor de abundância $>1 \%$, em um mínimo de duas amostras.

\section{Resultados \\ Composição e classificação taxonômica}

Foram identificadas 58 espécies (Tab. 2), representadas por 33 gêneros, com predominância de Gomphonema (9 spp.), Pinnularia (4 spp.), Thalassiosira (3 spp.), Cyclotella (3 spp.), Diploneis (3 spp.) e Placoneis (3 spp.).

\section{Riqueza de espécies}

A maior riqueza (34 spp.) ocorreu nas amostras 1 e 4 e a menor riqueza (23 spp.) na amostra 3 da transecção 1. Em média, a riqueza foi de 29 espécies. É importante observar que a riqueza esteve constituída predominantemente por espécies bentônicas e epifíticas, em detrimento das espécies planctônicas (Fig. 2).

\section{Abundância, freqüência de ocorrência e ecologia das espécies}

Entre as espécies numericamente mais representativas, $97,7 \%$ são de água doce e $8,3 \%$ são encontradas comumente nos estuários. Dentre as espécies identificadas, $82,75 \%$ são bentônicas/epifíticas e 17,25\% planctônicas, comuns em água doce a oligo-mesohalina. 
Tabela 2 - Composição e classificação das diatomáceas encontradas no sedimento superficial de intermaré da praia de Itupanema/Pará. Hab. = hábito; $\mathrm{b}=$ bentônico; $\mathrm{p}=$ planctônico; eps = epipsâmico; epf = epifítico.

\begin{tabular}{|c|c|}
\hline Táxons & Hab. \\
\hline \multicolumn{2}{|l|}{ BACILLARIOPHYTA } \\
\hline \multicolumn{2}{|l|}{ COSCINODISCOPHYCEAE } \\
\hline \multicolumn{2}{|l|}{ THALASSIOSIRALES } \\
\hline \multicolumn{2}{|l|}{ Thalassiosiracea } \\
\hline $\begin{array}{l}\text { Thalassiosira eccentrica } \\
\text { (Ehrenberg) Cleve }\end{array}$ & $\mathrm{p}$ \\
\hline T. oestrupii (Ostenfeld) Hasle & $\mathrm{p}$ \\
\hline Thalassiosira sp. & $\mathrm{p}$ \\
\hline $\begin{array}{l}\text { Tryblioptychus cocconeiformis } \\
\text { (Grunow ex Cleve) Hendey }\end{array}$ & b \\
\hline \multicolumn{2}{|l|}{ Stephanodiscaceae } \\
\hline Cyclotella meneghiniana Kützing & $\mathrm{p}$ \\
\hline C. striata (Kützing) Grunow & $\mathrm{p}$ \\
\hline C. stylorum Brightwell & $\mathrm{p}$ \\
\hline \multicolumn{2}{|l|}{ Heliopeltaceae } \\
\hline Polymyxus coronalis L. W. Bailey. & $\mathrm{p}$ \\
\hline \multicolumn{2}{|l|}{ BIDDULPHIOPHYCIDEA } \\
\hline \multicolumn{2}{|l|}{ BIDDULPHIALES } \\
\hline \multicolumn{2}{|l|}{ Biddulphiaceae } \\
\hline Terpsinoe musica Ehrenberg & $\mathrm{p}$ \\
\hline \multicolumn{2}{|l|}{ COSCINODISCOPHYCIDAE } \\
\hline \multicolumn{2}{|l|}{ PARALIALES } \\
\hline \multicolumn{2}{|l|}{ Paraliaceae } \\
\hline Paralia sulcata (Ehrenberg) Cleve & $\mathrm{p}$ \\
\hline \multicolumn{2}{|l|}{ AULACOSEIRALES } \\
\hline \multicolumn{2}{|l|}{ Aulacoseiraceae } \\
\hline \multicolumn{2}{|l|}{$\begin{array}{r}\text { Aulacoseira granulata } \\
\text { (Ehrenberg) Simonsen }\end{array}$} \\
\hline \multicolumn{2}{|l|}{ COSCINODISCALES } \\
\hline \multicolumn{2}{|l|}{ Coscinodiscaceae } \\
\hline $\begin{array}{l}\text { Actinocyclus normanii } \\
\text { (Gregory) Hustedt }\end{array}$ & $\mathrm{p}$ \\
\hline \multicolumn{2}{|l|}{ Heliopeltaceae } \\
\hline $\begin{array}{l}\text { Actinoptychus senarius } \\
\text { (Ehrenberg) Ehrenberg }\end{array}$ & $\mathrm{p}$ \\
\hline \multicolumn{2}{|l|}{ ACHNANTHALES } \\
\hline \multicolumn{2}{|l|}{ Achnanthaceae } \\
\hline $\begin{array}{l}\text { Achnanthes elata } \\
\text { (Leuduger-Fortmorel) Gandhi }\end{array}$ & $\mathrm{b}$ \\
\hline \multicolumn{2}{|l|}{ Cocconeidaceae } \\
\hline Cocconeis sp. & $\mathrm{b}$ \\
\hline \multicolumn{2}{|l|}{ Achnanthidiaceae } \\
\hline $\begin{array}{l}\text { Achnanthidium exiguum } \\
\text { (Grunow) Czarnecki }\end{array}$ & $\mathrm{b}$ \\
\hline
\end{tabular}

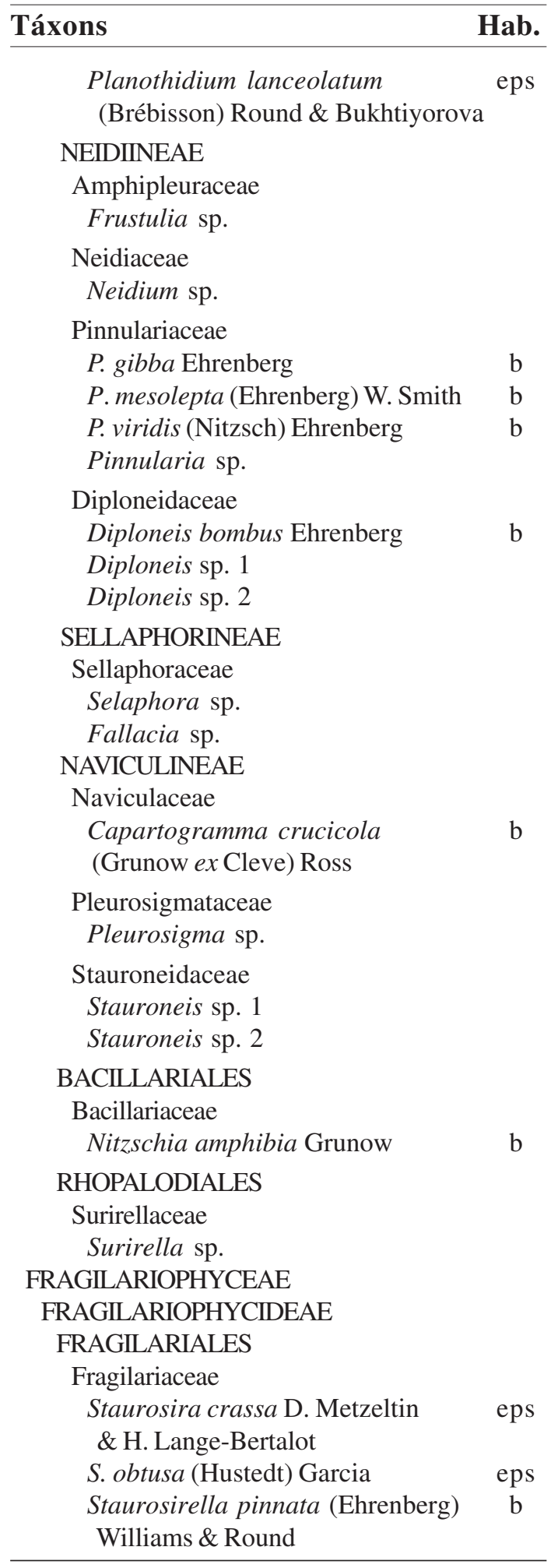




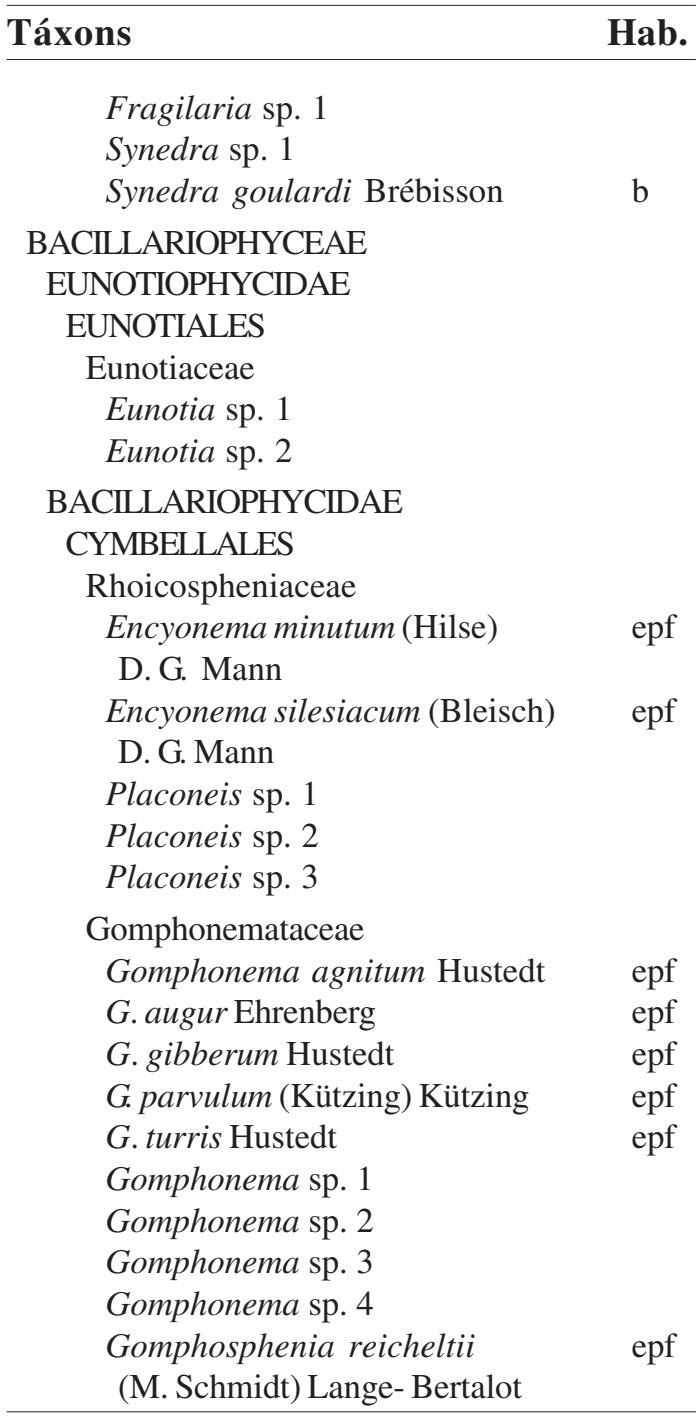

As espécies planctônicas Aulacoseira granulata, Cyclotella meneghiniana, Thalassiosira sp. e Thalassiosira eccentrica e as bentônicas/epifíticas Staurosirella pinnata, Encyonema silesiacum, Staurosira obtusa, Gomphonema parvulum, Planothidium lanceolatum, Encyonema minutum, Nitzschia amphibia e Frustulia sp. alcançaram conjuntamente, valores de abundância relativa e freqüência de ocorrência acima de $70 \%$, nas amostras analisadas (Figs. 3 e 4).

Entre as espécies muito freqüentes (100\%), ressalta-se a presença das espécies planctônicas Actinocyclus normanii e Polymyxus coronalis, entretanto, apresentaram baixos valores de abundância $(<3 \%)$. As espécies bentônicas/ epifíticas, Achnanthidium exiguum e Staurosirella crassa, com $90 \%$ de freqüência e Capartogramma crucicola e Gomphonema sp1, com $80 \%$ de freqüência, são pouco abundantes, não ultrapassando 4\% (Fig. 5).

Apenas A. granulata e Thalassiosira sp., foram espécies planctônicas abundantes, alcançando mais de $20 \%$. Entretanto, a primeira atingiu valor mínimo $(4,67 \%)$ na amostra arenosa 3 e valor máximo $(30,33 \%)$ na amostra argilosa 5. Thalassiosira sp. chegou a um mínimo de $1,67 \%$ na amostra arenosa 10 e ao máximo de 20,33\%, na amostra 5 .

A espécie bentônica/epifítica $S$. pinnata apresentou o maior percentual de abundância. As espécies bentônicas/epifíticas $S$. obtusa, E. sileciacum e E. minutum, Gomphonema parvulum, P. lanceolatum, N. amphibia e Frustulia sp., embora com menor representatividade de freqüência, atingiram valores importantes de abundância.

A diatomácea $S$. obtusa chegou ao valor máximo (10\%) na amostra 10 e mínimo $(1,67 \%)$ na amostra 6 (argilo-arenosa), enquanto $E$. silesiacum e E. minutum alcançaram os máximos de $11 \%$ e $7 \%$, respectivamente, nas amostras 8 e 4, ambas argilo-arenosas e mínimos de $0,33 \%$ na amostra 1 (arenosa) e $0,67 \%$ na amostra 5 (argilosa). Gomphonema parvulum atingiu abundância máxima na amostra 8 , com $7,67 \%$ e mínima na amostra 7 , com $1,67 \%$. P. lanceolatum atingiu o valor máximo de $11,33 \%$ na amostra arenosa 3 , sendo ausente nas amostras 4e 5. Nitzschia amphibia chegou a 4\% na amostra 3 e 1,67\% na amostra 4 e Frustulia sp., atingiu valor máximo de $9 \%$ na amostra 9 , foi ausente nas amostras 3, 4 e 8 .

As espécies planctônicas A. senarius e Paralia sulcata e as bentônicas Cocconeis sp., Tryblioptychus cocconeiformis, Diploneis sp. 2, D. bombus, Fragilaria sp. 2, Gomphonema agnitum, Gomphonema sp. 4, G. turris, Placoneis sp. 1, Pleurosigma sp., Surirella sp. e Terpsinoe musica ocorreram em apenas uma amostra (Fig. 5). 


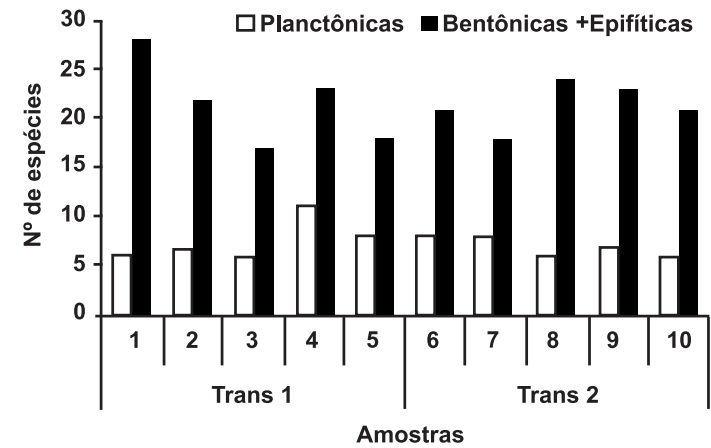

Figura 2 - Número de espécies (planctônicas e bentônicas/epifíticas) nas amostras de sedimentos superficiais da praia de Itupanema, Pará.

\section{Análises de agrupamentos das espécies}

$\mathrm{Na}$ análise de agrupamento, foram formados três grupos distintos, ao nível de corte de $67 \%$, relacionando-se provavelmente, com a textura dos sedimentos (Fig. 6). O grupo $1\left(\mathrm{G}_{1}\right)$ foi formado apenas pela amostra argilosa 5. O grupo $2\left(\mathrm{G}_{2}\right)$, foi formado pelas amostras 1, 2, 4, 6, 7, 8 e 9 , que apresentam composição sedimentar variando de arenosa a argilo-arenosa. $\mathrm{O}$ grupo $3\left(\mathrm{G}_{3}\right)$ foi formado pelas amostras arenosas 3 e 10 .

A Análise de Correspondência (AC), mostrou um padrão semelhante ao observado na análise de agrupamento Bray-Curtis, porém com um refinamento maior no padrão de distribuição das amostras/espécies, onde os dois primeiros eixos explicaram 50,97\% da variância total com seus respectivos "eigenvalues" (Tab. 3).

Nesta análise, o eixo 1 com $31 \%$ da variância explicada, situou a amostra argilosa 5 isolada à esquerda, associada com os táxons planctônicos A. granulata e Thalassiosira sp. As amostras 1, 2, 4, 6, 7, 8 e 9 foram situadas no centro do diagrama, notando-se que a maioria das espécies são encontradas nessas amostras, entretanto há maior similaridade entre as amostras argilo-arenosas 2, 4, 6, 7 e 9, distanciando-se das amostras 1 (arenosa) e 8 (argilo-arenosa). Por fim, as amostras arenosas 3 e 10 estão situadas à direita, associadas com $S$. pinnata e $P$. lanceolatum, bentônicas/epifíticas.

O eixo 2 explica 19,949\% da variância, mostrando um principal agrupamento das amostras 2, 4, 6, 7 e 8, situadas no centro do diagrama. Entretanto, há um outro grupo, mais
Tabela 3- "Eingenvalues" e variância explicada encontrados na AC das espécies nas amostras superficiais da praia de Itupanema, Pará.

\begin{tabular}{lcc}
\hline Eixos & Eingenvalues & Variância explicada (\%) \\
\hline Eixo 1 & 0.14455 & 31.023 \\
Eixo 2 & 0.092951 & 19.949 \\
Eixo 3 & 0.0851132 & 18.267 \\
Eixo 4 & 0.0533951 & 11.459 \\
\hline
\end{tabular}

distante do grupo anterior, situado um pouco acima deste, formado pela amostra 1 (arenosa) ligado às espécies bentônicas/epifíticas Cocconeis sp., Diploneis sp. 1, Stauroneis sp. 1 e Tryblioptchus cocconeiformis e amostra 9 (argilo-arenosa), associada com Frustulia sp., bentônica/epifítica.

\section{Discussão}

Os valores de abundância relativa e distribuição das diatomáceas da praia de Itupanema parecem estar fortemente ligados à textura dos sedimentos do sistema estuarino. Sedimentos argilosos evidenciam um ambiente de sedimentação de menor energia e portanto, favoreceram a deposição de táxons planctônicos, enquanto sedimentos arenosos, de ambiente de sedimentação com maior energia, mostraram a dominância de táxons bentônicos.

Nos sedimentos superficiais de Itupanema, embora as espécies planctônicas estivessem pouco representadas em termos de riqueza de espécies, apresentaram os maiores valores de abundância nos sedimentos argilosos. As espécies provenientes da coluna d'água, rica em sedimentos em suspensão, que foram depositados conjuntamente nesses ambientes mais calmos, estariam menos sujeitas aos processos de abrasão e dissolução, que normalmente ocorrem nas zonas de intermaré, em condições de maior energia (Mitbavkar \& Anil 2002).

As espécies de diatomáceas encontradas no fitoplâncton do rio Pará, que banha a praia de Itupanema (El-Robrini et al. 2006), quando comparadas com as espécies encontradas no sedimento do ambiente praial, mostraram um número muito baixo de espécies planctônicas, 

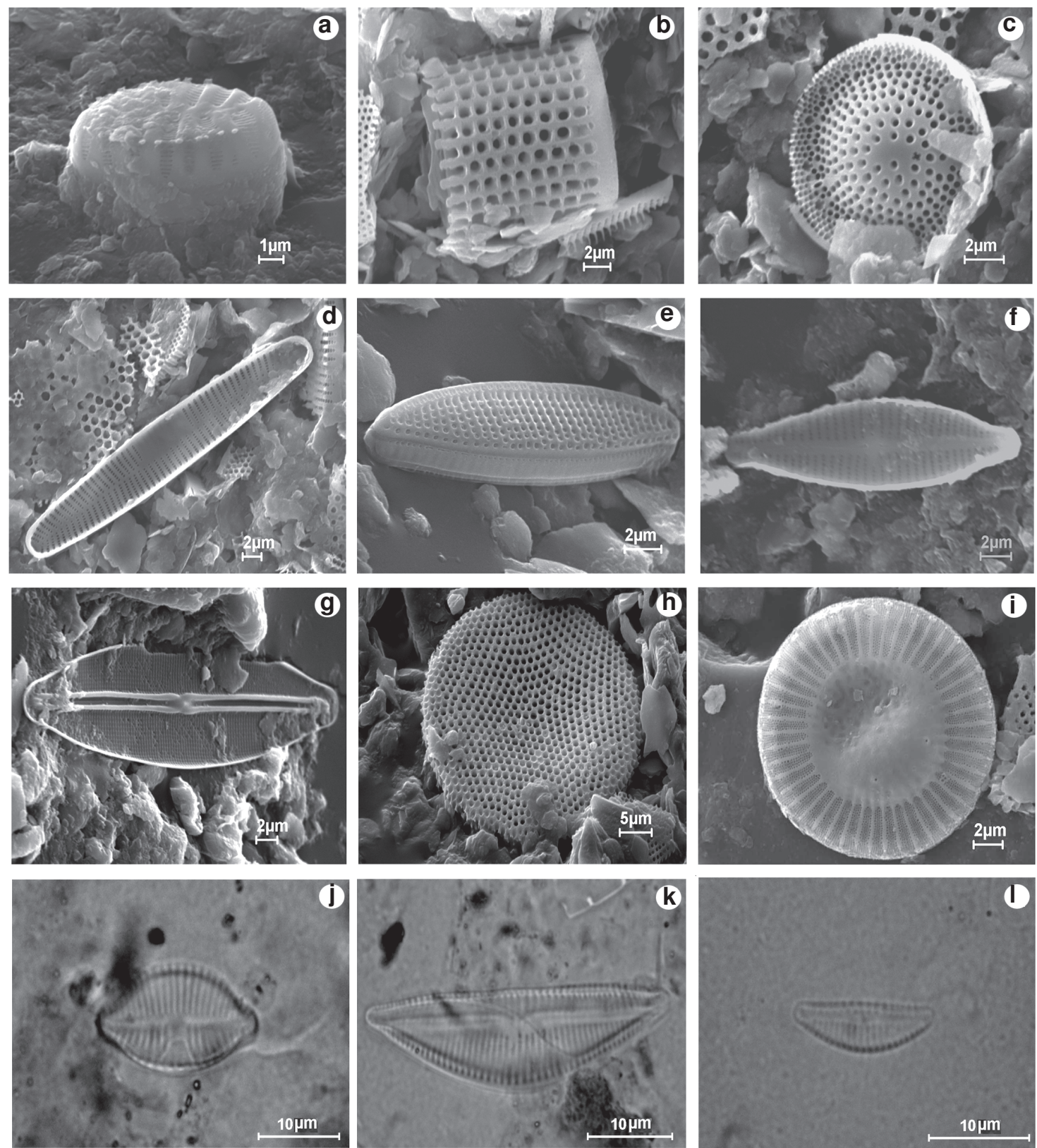

Figura 3 - Espécies numericamente mais representativas encontradas nas amostras de sedimento superficial da praia de Itupanema/PA. a. Staurosirella pinnata; b. Aulacoseira granulata; c. Thalassiosira sp.; d. Staurosira obtusa; e. Nitzschia amphibia; f. Gomphonema parvulum; g. Frustulia sp.; h. T. eccentrica; i. Cyclotella meneghiniana (Microscopia eletrônica de varredura); j. Planothidium lanceolatum; k. Encyonema silesiacum; 1. Encyonema minutum (Microscopia de luz).

onde apenas as espécies planctônicas limnéticas A. granulata e as marinho/estuarinas $P$. coronalis e C. stylorum foram comuns aos dois ambientes (água e sedimentos).

Os trabalhos de fitoplâncton na baía do Guajará realizados por Moreira-Filho et al. (1974) e Paiva et al. (2006), mostraram um aumento no número de espécies, se comparados ao trabalho de El-Robrini et al. (2006). Entretanto, os valores de abundância permaneceram baixos, em comparação aos sedimentos da praia de Itupanema, onde seis espécies foram comuns às encontradas por Paiva et al. (2006): A. granulata, A. senarius, $C$. stylorum, T. eccentrica, $P$. 

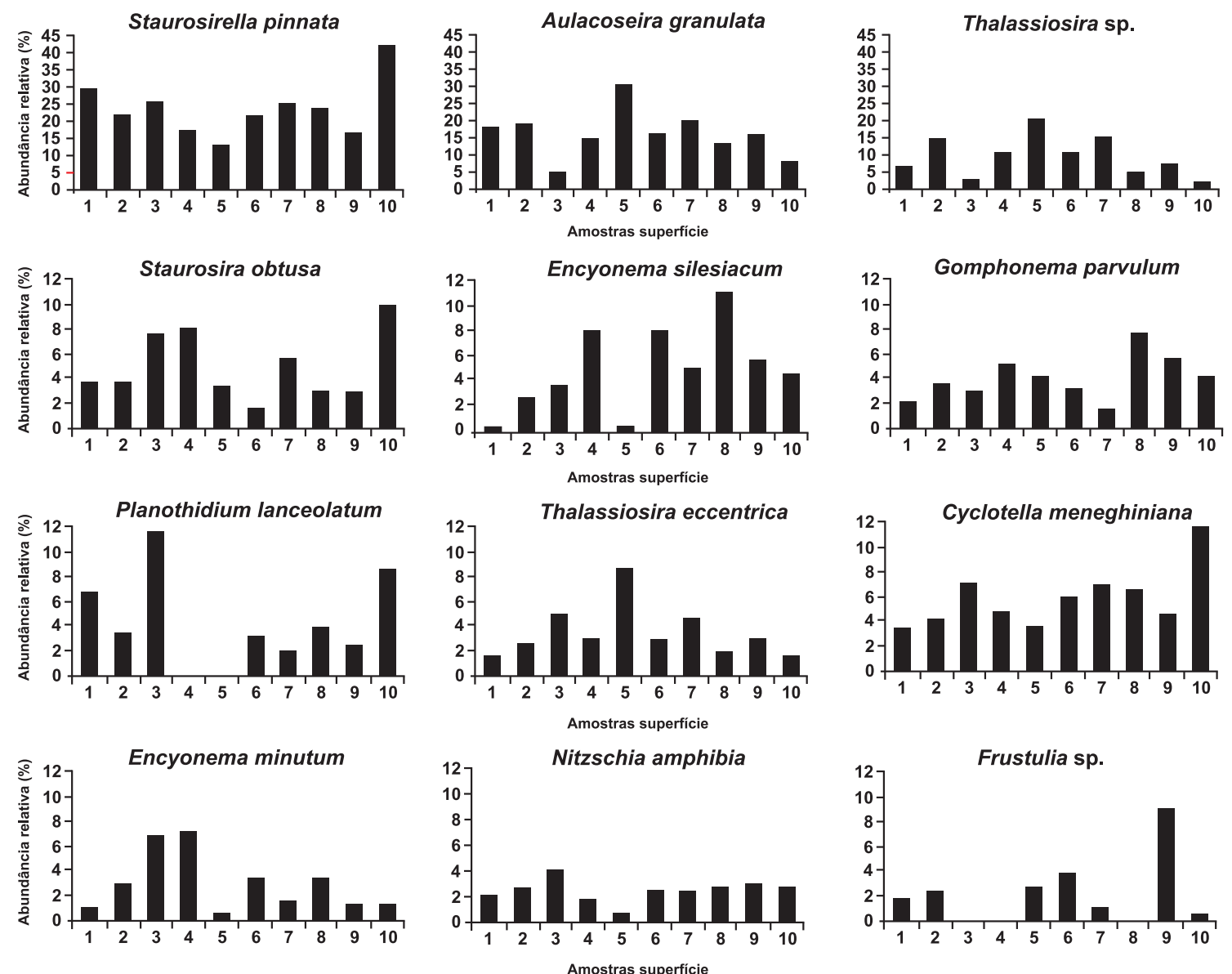

Figura 4 - Abundância relativa das espécies numericamente mais representativas, ao longo das amostras.

coronalis e T. musica. E outras seis foram comuns às encontradas por Moreira-Filho et al. (1974): A. normanii, A. senarius, C. meneghiniana, C. stylorum, A. granulata e $P$. coronalis.

A praia de Itupanema mostrou-se um ambiente desfavorável à sedimentação de espécies provenientes da coluna d'água, exemplificado pela espécie Polymyxus coronalis, encontrada no fitoplâncton da região com elevados valores de abundância, em torno de $50 \%$ (Paiva et al. 2006) e 30\% (El Robrini et al. 2006), porém sua abundância não excedeu $2 \%$ nos sedimentos superficiais da área de estudo.

Destacaram-se as espécies planctônicas Aulacoseira granulata e Thalassiosira sp., pois ambas obtiveram maiores valores de abundância na amostra 5, argilosa, sendo responsáveis, conjuntamente, por $50,66 \%$ da abundância total.
No fitoplâncton do rio Pará, A. granulata ocorreu com valores de abundância em torno de $10 \%$ (El Robrini et al. 2006), enquanto nos sedimentos de Itupanema, com exceção das amostras arenosas, todas as abundâncias superaram este valor, alcançando o máximo de $30 \%$ na amostra 5, argilosa. Vélez \& Hooghiemstra (2005) reportaram esta espécie com maiores percentuais em raízes da zona litoral $(36,17 \%)$ do que em sedimentos lacustres de fundo $(22,89 \%)$ e no plâncton (23,85\%), no nordeste da Colômbia.

Assim, as altas abundâncias de $A$. granulata nos sedimentos superficiais de Itupanema, poderiam relacionar-se à resistência de sua frústula aos processos de abrasão e dissolução em ambientes turbulentos, uma vez que é fortemente silicificada (Bradbury 2000; Vélez \& Hooghienmstra 2005; O‘Farrel et al. 2001; Gomes et al. 2003). 
Frequência de ocorrência (\%)
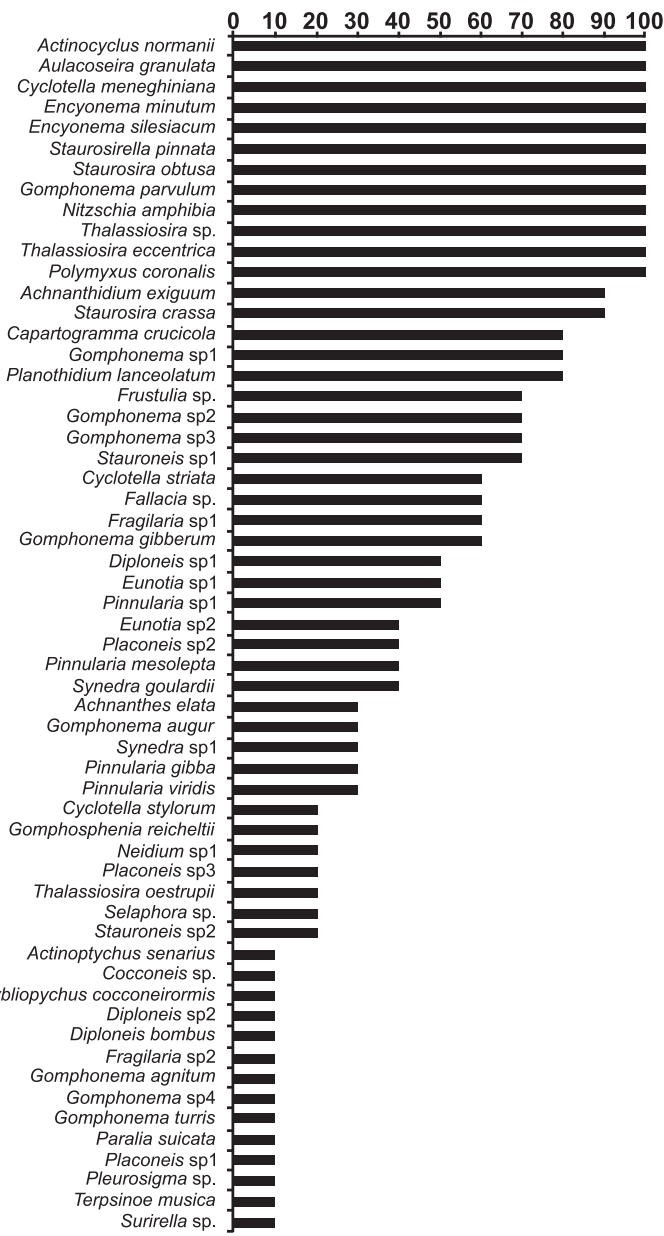

Figura 5 - Frequiência de ocorrência das espécies em sedimentos superficiais da praia de Itupanema/Pará.

O gênero Thalassiosira é preferencialmente marinho, todavia, ocorrem algumas espécies em água doce (Aké-Castillo et al. 1999). Não há informações ecológicas sobre esse táxon, uma vez que este não foi referenciado em outros trabalhos para a região. Entretanto, o mesmo foi muito freqüente e abundante na área de estudo, apresentando valores semelhantes aos de $A$. granulata, merecendo estudos mais detalhados à respeito de sua taxonomia e ecologia.

Na praia de Itupanema, as diatomáceas planctônicas foram suplantadas pelas bentônicas em número de espécies. Entretanto, as bentônicas apresentaram baixos valores de abundância. O hábito destes fitobentos incluiria a vegetação existente na área de coleta, além de sedimentos arenosos e argilosos.

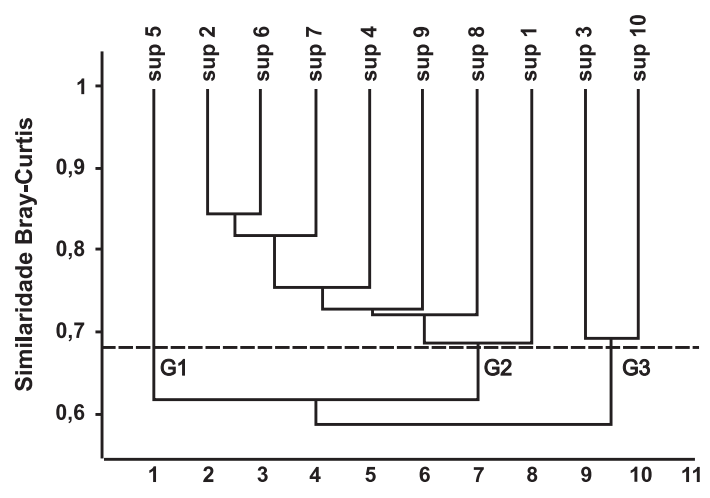

Figura 6 - Análise de agrupamento das amostras de sedimentos superficiais da praia de Itupanema/Pará.

Os altos valores de abundância de Staurosirella pinnata, em sedimentos superficias, foi evidenciada em diversos estudos, porém com diferentes explicações para sua ocorrência. Round et al. (1990) consideraram o gênero Staurosirella limnético, ocorrendo muitas vezes aderido aos grãos de areia. Gomes et al. (2005), na análise dos sedimentos superficiais da lagoa do Boqueirão-RN, mostraram que a diatomácea perifítica $S$. pinnata ocorreu como uma das espécies mais abundantes $(41,06 \%)$, caracterizando os trechos mais rasos do sistema, associada ao banco de macrófitas existente. A assembléia de sedimentos superficiais da lagoa hipersalina Araruama, RJ, mostrou a dominância de Staurosira elliptica (Schumann) Williams \& Round e Staurosirella pinnata, em três áreas da lagoa, sujeitas ao aporte de água proveniente de rios (Sylvestre et al. 2001). A espécie epipsâmica S. elliptica formou colônias em forma de fita, enquanto $S$. pinnata, considerada ticoplanctônica, foi observada em água doce a oligosalina (0-5 de salinidade), associadas às zonas com forte variação na profundidade (Sylvestre et al. 2001). Hassan et al. (2006) encontraram as espécies ticoplanctônicas Staurosira construens var. venter e Staurosirella pinnata dominando o interior da lagoa Mar Chiquita, Argentina, cujas condições ambientais eram bastante variáveis, em água salobra/doce e com altas concentrações de sedimentos em suspensão. Vale ressaltar que, onde as duas espécies foram mais abundantes, o tipo de sedimento era grosseiro, variando de areia grossa com conchas a areia grossa com silte. 
Entretanto, houve uma variabilidade muito grande dos ambientes em relação à distribuição de $S$. pinnata, assim como diferentes formas de vida (perifíticas, ticoplanctônicas e epipsâmicas), necessitando-se portanto de estudos mais aprofundados acerca de sua ecologia e distribuição geográfica.

As assembléias de diatomáceas em sedimentos superficiais de lagoas rasas do SE da Inglaterra, enriquecidas artificialmente, mostraram dominância de Fragilaria spp., gênero subdividido em diversos outros gêneros por Williams \& Round (1987), entre eles Staurosira e Starousirella, muitas destas associadas ao hábito bentônico (Bennion 1995). Sua dominância em sedimentos superficiais pode ser explicada pela sua associação à zona litoral de águas rasas, o que proveria um espaço considerável para a colonização de formas bentônicas, uma vez que esses sedimentos fazem parte da zona fótica.

As macrófitas aquáticas afetam a composição, tamanho de partícula e a distribuição local dos sedimentos em ambientes costeiros, reduzindo a energia das ondas e a velocidade das correntes. Esta redução não somente aumenta as taxas de sedimentação, como diminui o potencial para resuspensão, reduzindo também a turbideze aumentando assim a disponibilidade de luz, o que promove o crescimento e a reprodução das plantas aquáticas (Doyle 2000, apud Madsen et al. 2001).

Em áreas costeiras, os sedimentos finos acumulam-se em áreas colonizadas por macrófitas aquáticas. Uma vez que a biomassa dessas plantas aumenta a sedimentação, as áreas com baixa densidade de indivíduos apresentam baixa sedimentação, ou mesmo resuspensão ativa, enquanto bancos densos são áreas de alta sedimentação.

Desta forma, além de servir de substrato para a colonização das diatomáceas epifíticas, a vegetação aquática seria um dos fatores responsáveis pela variação textural dos sedimentos, uma vez que reduzem a velocidade de corrente e a energia das ondas em áreas

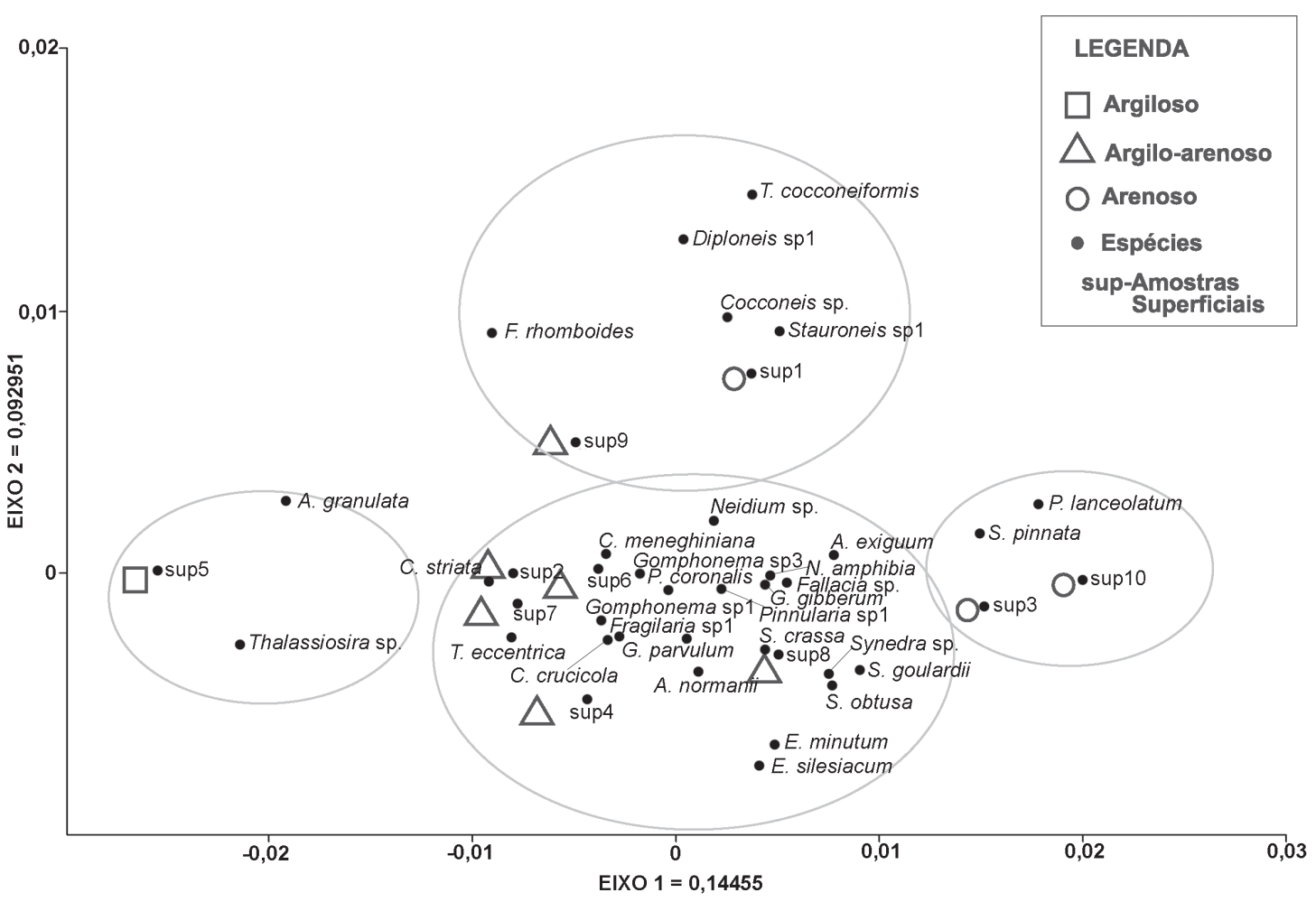

Figura 7 - Análise de correspondência mostrando quatro grupos de diatomáceas associados às diferentes texturas das amostras sedimentares superficiais da praia de Itupanema/Pará. 
costeiras (Madsen et al. 2001), promovendo a retenção de matéria particulada fina em suas folhas (Pluntke \& Kozerski 2003).

Assim, diferentes densidades da vegetação no campo herbáceo, poderiam produzir, localmente, diferentes taxas de sedimentação, onde em zonas mais densas, predominariam sedimentos argilosos, obdecendo um gradiente com sedimentos argilo-arenosos até zonas sem vegetação com sedimentos arenosos.

\section{Conclusões}

Os resultados mostraram a influência da relação areia/argila sobre a composição das diatomáceas, que pode explicar a prevalência de agrupamentos de espécies ora planctônicas, ora bentônicas na zona intermaré da praia de Itupanema/PA.

Os sedimentos argilosos evidenciaram um ambiente de sedimentação fina de menor energia, favorecendo a deposição e preservação de diatomáceas planctônicas Aulacoseira granulata e Thalassiosira sp., enquanto sedimentos arenosos predominaram em um ambiente com maior energia, prevalecendo as diatomáceas bentônicas Staurosirella pinnata, Staurosira obtusa e Planothidium lanceolatum.

Os sedimentos argilo-arenosos estiveram associadas tanto a comunidades de diatomáceas planctônicas, quanto diatomáceas bentônicas.

Há de se salientar a contribuição da vegetação herbácea da zona intermaré da praia de Itupanema, atenuando a energia das ondas e marés, retendo os sedimentos finos argilosos, parecendo exercer assim, uma forte influência na sedimentação, promovendo a redução de processos de abrasão na zona intermaré e a preservação dos táxons planctônicos.

\section{Agradecimentos}

Aos pesquisadores Dra. Regina Célia Tavares Lobato do Museu Parense Emílio Goeldi e Dr. Rosildo Santos Paiva da Universidade Federal do Pará pelas críticas ao manuscrito; ao pesquisador MSc. Paulo Roberto do Canto Lopes e à Companhia Vale do Rio Doce pelo financiamento à pesquisa e ao $\mathrm{CNPq}$ pela concessão de bolsa de Mestrado ao primeiro autor.

\section{REFERÊNCIAS BIBLIOGRÁFICAS}

Ab' Saber, A. N. 2006. Brasil: paisagens de exceção: o litoral e o Pantanal MatoGrossense: patrimônios básicos. Ateliê Editorial, Cotia, 182p.

Aké-Castillo, J. A.; Hernández-Becerril, D. U. \& Castillo, M. E. M. 1999. Species of the genus Thalassiosira (Bacillariophyceae) from the Gulf of Tehuantepec, Mexico. Botanica Marina 42: 487-503.

Alves, M. A. M. S; El-Robrini, M.; Sousa-Filho, P. W. M; Farias, D. R. \& França, C. F. 2005. Morfodinâmica das praias de mesomacromarés da zona costeira do estado do Pará. In: X Congresso da ABEQUA Associação Brasileira de Estudos do Quaternário, Guarapari, ES. CD-ROM de resumos expandidos, $\mathrm{n}^{\circ} 0258$.

Bennion, H. 1995. Surface-sediment diatom assemblages in shallow, artificial, enriched ponds, and implications for reconstructing trophic status. Diatom Research 10(1): 1-19.

Bradbury, J. P. 2000. Limnologic history of Lago de Pátzcuaro, Michoacán, Mexico for the past 48,000 years: impacts of climate and man. Palaeogeography, Palaeoclimatology, Palaeoecology 163: 69-95.

Burkholder, J. M. 1996. Interactions of benthic algae with their substrata. In: Stevenson, R. J.; Bothwell, M.I. \& Lowe, R. L. (eds.). Algal ecology: freshwater benthic ecosystems. Academic Press, Inc. Pp. 253-297.

Callegaro, V.L. \& Lobo, E. A. 1990. Distribuição horizontal da comunidade de diatomáceas em turfeiras holocênicas da planície costeira do Rio Grande do Sul, Brasil. Caderno de Pesquisa, Série Botânica 2(1): 5-22

Cooper, S. R. 1995. Diatoms in sediment cores from mesohaline Chesapeake Bay, U. S. A. Diatom Research 10: 39-89.

1999. Estuarine palaeoenvironmental reconstructions using diatoms. In: Stoermer, E. F. \& Smol, J. P. (eds.). The Diatoms: Applications for the environmental and sciences. University Press, Cambridge. Pp. 352-373.

El-Robrini, M.; Melo, N. F. A. C. \& Santos, M.L. 2006. Resultados das análises de qualidade 
de água, sedimentos de fundo, comunidades aquáticas (plâncton, bentos \& nécton) e bacteriologia no rio Pará, nas imediações do futuro terminal portuário graneleiro de Barcarena (porto de Vila do Conde, Barcarena-PA) (1 ${ }^{\mathrm{a}}$ campanha). Relatório Técnico. Universidade Federal do Pará, Belém, 75p.

Felício-Fernandes, G. \& Souza-Mosimann, R. M. 1994. Diatomáceas no sedimento do manguezal de Itacorubi-Florianópolis, Santa Catarina, Brasil. Insula, Florianópolis 23: 149-215.

Fritz, S. C.; Cumming, B. F.; Gasse, F. \& Laird, K. R. 1999. Diatoms as indicators of hydrologic and climatic change in saline. In: Stoermer, E. F. \& Smol, J. P (eds.). The Diatoms: Applications for the environmental and sciences. University Press, Cambridge. Pp. 41-72.

Garcia-Baptista, M. 1996. The distribution of psammic algae on a marine beach at Praia Azul, Brazil. In: Frank E. Round. (org.). Proceedings of the Thirtieth Diatom Symposium. Bristol: Biopress. 13: 183-205.

Garcia, M. 2006. The transfer of Fragilaria obtusa Hustedt to the genus Staurosira Ehrenberg (Bacillariophyceae). Phycological Research 54(2): 87-93.

Gomes, D. F.; Brichta, A.; Silva, E. M.; Fernandes, L. F. 2003. Diatomáceas como indicadoras de mudanças ambientais da Baía de Iguape, Baixo Paraguaçu (Bahia, Brasil). In: IX Congresso da ABEQUA - Associação de Estudos do Quaternário. CD-ROM de resumos expandidos, $\mathrm{n}^{\circ} 168$.

Gomes, D. F.; Albuquerque, A. L. S.; Sifeddine, A. \& Turcq, B. 2005. Heterogeneidade espacial da comunidade de diatomáceas nos sedimentos superficiais da lagoa do Boqueirão (RN) como fator de calibração para interpretações paleolimnológicas. In: X Congresso da ABEQUA - Associação Brasileira de Estudos do Quaternário. CDROM de resumos expandidos $n^{\circ} 0208$.

Hall, R. I. \& Smol, J. P. 1999. Diatoms as indicators of lake eutrophication. In: Stoermer, E. F. \& Smol, J.P. (eds.). The Diatoms: Applications for the environmental and sciences. University Press, Cambridge. Pp. 352-373.

Hassan, G. S.; Espinosa, M. A. \& Isla, F. I. 2006. Modern diatom assemblages in surface sediments from estuarine systems in the southeastern Buenos Aires Province, Argentina. Journal of Paleolimnology 35: 39-53.

Hendey, N. 1964. An introductory account of the smaller algae of British coastal waters - V.Bacillariophyceae (Diatoms). Fischery Investigations Series, London: Her Majesty's Stationery Office 4(5): 317p.

Lemos, R. C. \& Santos, R. D. 1996. Manual de descrição e coleta de solo no campo. $3^{\mathrm{a}}$ ed. Sociedade Brasileira de Ciência do Solo, Campinas, 83p.

Lopes, P. C. 2005. Programa de arqueologia preventiva na área do projeto Bauxita Paragominas/Pa. Relatório de escavação do sítio arqueológico PA-BA-84: ALUNORTE. Museu Paraense Emílio Goeldi, Belém, 64p.

Madsen, J. D.; Chambers, P. A.; James, W. F.; Koch, E. W. \& Westlake, D. F. 2001. The interaction between water movement, sediment dynamics and submersed macrophytes. Hydrobiologia 444: 71-84.

Margalef, R. 1958. Temporal succession and spatial heterogeneity in phytoplankton. In: Buzzati-Traverso, A. A. (ed.). Pespectives in marine biology. University of California Press, Berkeley. Pp. 323-349.

Martínez de Fabricius, A. L; Maidana, N.; Gómez, N. \& Sabater, S. 2003. Distribution patterns of benthic diatoms in a Pampean river exposed to seasonal floods: the Cuarto River (Argentina). Biodiversity and Conservation 12: 2443-2454.

Mitbavkar, S. \& Anil. A.C. 2002. Diatoms of the microphytobenthic community: population structure in a tropical intertidal sand flat. Marine Biology 140: 41-57. 2006. Diatoms of the microphytobenthic community in a tropical intertidal sand flat influenced by monsoons: spatial and temporal variations. Marine Biology 148: 693-709. 
Moreira Filho, H.; Valente-Moreira, I. M. \& Trippia-Cecy. I. I. 1974. Diatomáceas do rio Guamá (foz do rio-Belém-estado do Pará). Leandra 4-5: 123-135.

Moreira-Filho H.; Valente-Moreira I. M.; SouzaMosimann R. M. \& Cunha J. A. 1990. Avaliação florística e ecológica das diatomáceas (Chrysophyta-Bacillariophyceae) marinhas e estuarinas nos estados do Paraná, Santa Catarina e Rio Grande do Sul. Estudos de Biologia 25: 5-48.

Moro, R. S. \& Fürstenberger, C. B. 1997. Catálogo dos principais parâmetros ecológicos de diatomáceas não-marinhas. Ed. UEPG, Ponta Grossa, 282p.

Moro, R. S. \& Santi, V. 1999. Avaliação das técnicas de oxidação comumente empregadas na limpeza de valvas silicosas. Boletim da Sociedade Brasileira de Limnologia 26: 17-25.

Munsell Colors Company. 1975. Munsell Soil Coors Charts. Baltimore, Maryland USA.

O'Farrell, I.; Tell, G. \& Podlejski, A. 2001. Morphological variability of Aulacoseira granulata (Ehrenberg) Simonsen (Ehr.) Simonsen (Bacillariophyceae) in the Lower Paraná River (Argentina). Limnology 2: 65-71.

Oliveira, R, D. 2002. Zoneamento agroambiental do Município de Barcarena-Pará. Dissertação de Mestrado. Faculdade de Ciências Agrárias do Pará, 110p.

Paiva, R. S.; Eskinazi-Leça, E.; Passavante, J. Z. O.; Silva-Cunha, M. G. G. \& Melo, N. F. A. C. 2006. Considerações ecológicas sobre o fitoplâncton da Baía do Guajará e foz do rio Guamá (Pará-Brasil). Boletim do Museu Paraense Emílio Goeldi, Série Ciências Naturais. n.2, 2:133-146.

Patrick, R.; Reimer, C. W. 1966. The Diatoms of United States: exclusive of Alaska and Hawaii. Philadelphia: Academy of Natural Sciences 1(13): 688.

Pielou, E. C. 1969. An introduction to mathematical ecology. Wiley, New York, 286p.

Pires, L. C. \& Lacerda, L. D. 2004. Diatomáceas em biofilme da interface sedimento-água no Manguezal de Coroa Grande, Baía de Sapetiba, RJ. Arquivos de Ciências do Mar 37: 105-111.

Pluntke, T. \& Koserski, H. P. 2003. Particle trapping on leaves and on the bottom in simulated submerged plants stands. Hydrobiologia 506-509: 575-581.

Ribeiro, F. C. P. 2007. Palinomorfos aquáticos (diatomáceas) em sedimentos lamosos de intermaré da praia de Itupanema, município de Barcarena, Pará. Dissertação de Mestrado. Universidade Federal Rural da Amazônia, 76p.

Ribeiro, F. C. P.; Senna, C. S. F. \& Costa, B. O. 2007. Análise de diatomáceas e relação água doce/água salgada na planície costeira de Soure /PA. In: Anais do XI Congresso aa Associação Brasileira de Estudos do Quaternário - Abequa. CDROM de resumos expandidos.

Round, F. E.; Crawford, R. M.; Mann, D. G. 1990. The Diatoms: biology and morphology of the genera. Cambridge University Press, Cambridge, $747 \mathrm{p}$.

Silva, R. L. \& Cimardi, J. M. 1989. Nota sobre a utilização do "trapping method" no estudo das diatomáceas epipélicas do manguezal de Ratones, Florianópolis, SC. Insula 19(Supp.): 299-304

Sylvestre. F.; Beck-Eichler, B.; Duleba, W. \& Debenay, J. P. 2001. Modern benthic diatom distribution in a hypersaline coastal lagoon: the Lagoa de Araruama (RJ), Brazil. Hydrobiologia 443: 213-231.

Vélez, M. I. \& Hooghiemstra. 2005. Fossil and modern diatom assemblages from the savanna lake El Piñal, Colombia: An environmental reconstruction. Diatom Research 20(2): 387-407.

Vos, P. C. \& De Wolf, H. 1993. Diatoms as a tool for reconstructing sedimentary environments in coastal wetlands; methodological aspects. Hydrobiologia 269/270: 285-296.

Williams, D. M. \& Round, F. E. 1987. Revision of the genus Fragilaria. Diatom Research 2(2): 267-288. 